\title{
Comparing different models and response transformations in an information integration task
}

\author{
IRWIN P. LEVIN \\ University of Iowa, Iowa City, Iowa 52242
}

\begin{abstract}
The role of response transformations was examined in an information integration task in which different models were supported for different response scales. In Experiment 1, one group of subjects made subjective estimates of cost in dollars (cost estimates) for a series of hypothetical trips described by varying levels of gasoline price, gas mileage, and distance and another group of subjects made comparative ratings of the relative expense of each trip. A multiplicative model was supported for the cost estimates and an additive model was supported for the comparative ratings. In Experiment 2, a single group of subjects made cost estimates and then were asked, in the absence of stimulus values, to convert these judgments to comparative ratings. A multiplicative model was supported for each response mode, indicating a linear response transformation. It was concluded that the different response patterns in Experiment 1 were due to different integration processes for different response requirements rather than a nonlinear response transformation.
\end{abstract}

Information integration theory (Anderson, 1974) attempts to describe complex cognitive processes with simple algebraic models and has been applied to such diverse tasks as personality impression formation (Anderson, 1962) and consumer decisions (Levin, 1975). One problem in defining the underlying cognitive process is that the resultant of the internal integration process must undergo an unobservable transformation to the required response scale. If the response transformation is linear, then the picture gotten from the raw data parallels the underlying integration process; otherwise it does not.

The present study illustrates a new method for examining whether the model describing the overt responses accurately portrays the underlying integration process. Previous investigators have used a cross-task validity test where different tasks (e.g., averaging and difference) are used, and therefore different models are applicable, but the stimulus values and response scale are the same. If subjective scales are invariant (within linear transformations), and if simple models fit the data for the various tasks, then joint support is taken for the validity of the response scale and the integration models (Anderson, 1972; Birnbaum, 1974; Birnbaum \& Veit, 1974; Levin, 1975). By contrast, the present study examines two variants of an integration task using the same stimuli, the same "rational" model, but different response scales. In Experiment 1, the comparison was conducted across different groups of subjects where an inferred response transformation was one possible explanation of why the pattern of results differed

The author would like to thank Valerie Hensley and Fernando Corry for their help in collecting and compiling the data. between the two tasks. In Experiment 2, a within-subjects comparison was made where any response transformation could be observed directly.

\section{TASKS}

In each experiment, the subjects were required to make subjective judgments of travel expense for hypothetical trips described by varying levels of gasoline price $(P=40,60$, or 80 cents $)$, expected average gasoline mileage $(M=14,21$, or $28 \mathrm{mpg})$ and distance to be travelled $(\mathrm{D}=1,000,1,500$, or 2,000 miles). Each subject received three replications of the 27 possible combinations of the three factors, with the first replication serving as practice. The "rational" model for combining these three categories of information to obtain accurate estimates of travel cost is $\mathrm{P}$ by $\mathrm{D}$ by $\mathrm{M}^{-1}$.

The specific tasks used for the different experimental groups are explained in detail by Hensley and Levin (Note 1). Briefly, one task-cost estimates - required subjects to give their subjective or intuitive estimate of the cost in dollars of each hypothetical trip. The other task-comparative ratings-required subjects to indicate on a line-mark scale (later converted to a 20-point scale) how expensive or inexpensive each trip seemed in comparison to the other trips. In order to permit comparative ratings, subjects in this task were shown a summary sheet describing all the trips before they started their ratings.

In Experiment 1 , one group $(n=12)$ received the cost estimates task, and another group $(n=21)$ received the comparative ratings task. In Experiment 2 , a single group $(n=21)$ received both tasks. 


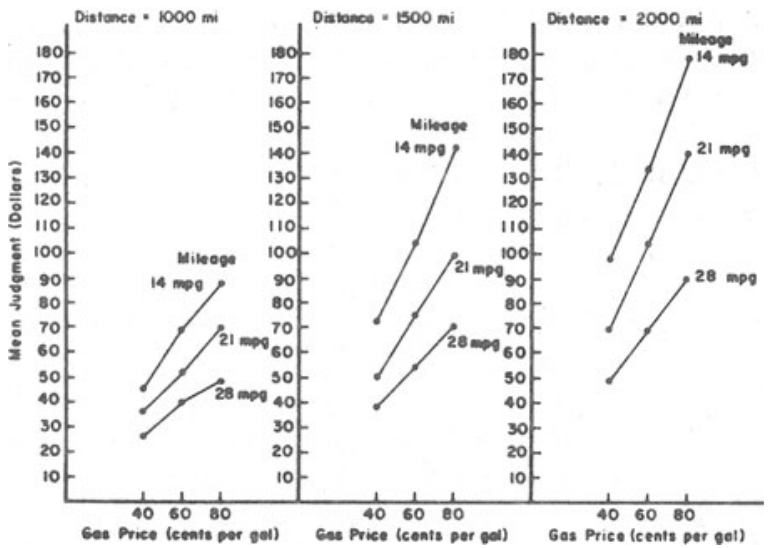

Figure 1. Mean numerical Judgments of travel cost for Experiment 1.

These subjects initially estimated the cost in dollars of each trip. Then the stimulus materials were removed and, referring only to their answer sheets, they were required to convert their dollar estimates into comparative ratings.

\section{RESULTS AND DISCUSSION}

\section{Experiment 1}

The results for Experiment 1 are summarized in Figures 1 and 2. Mean responses for the cost estimates group are shown in Figure 1 and exhibit the pattern of results predicted by a multiplying model. This model predicts a diverging fan of straight lines for each panel, reflecting interaction effects that are concentrated in the linear components. The statistical analysis (see Graesser \& Anderson, 1974, Appendix) supported a multiplying model for the group data and for the majority of individual subjects, although individuals differed in their mean rating and in the relative influence of each factor. The most appropriate model for describing these data is the following subjective analogue of the rational model:

$$
R=d^{w} \mathbf{D} \times p^{w} \mathbf{P} \times m^{-w} \mathbf{M}
$$

where $d, p$, and $m$ represent the subjective correspondents of stimulus values of $\mathrm{D}, \mathrm{P}$, and $\mathrm{M}$, and $w_{D}, w_{P}$, and $w_{M}$ represent the weights of the different categories of information.

Mean ratings of the comparative rating group are shown in Figure 2 and exhibit the pattern of results predicted by an additive model. The lines in each panel. rather than diverging, are approximately parallel. None of the two-way interactions approached statistical significance and the three-way interaction, $D$ by $P$ by $M$, was of borderline significance at the .05 level due to a slight convergence of the lines in the left-hand panel of Figure 2. An additive model of the following form closely approximates the data:

$$
R=w_{D} d+w_{P} p-w_{M} m
$$

where the symbols are as defined in Equation 1.

\section{Experiment 2}

Experiment 2 was designed to compare the following two interpretations of the difference between the cost estimates and relative ratings of Experiment 1: (1) The information might have been integrated via a multiplicative process in each case but the ratings were subject to a nonlinear (logarithmic) transformation that led to additive data. (2) The information might have been integrated by a different process in each case where the overt responses directly reflect the underlying process. The subjects in Experiment 2 were required to make an overt transformation of cost estimates to relative ratings.

Figure 3 summarizes the cost estimates and Figure 4 summarizes the relative ratings. It can be seen that the pattern of results is similar for the cost estimates of Experiment 2 (Figure 3) and the cost estimates of Experiment 1 (Figure 1). The dotted lines in Figure 3 give numerically correct values of travel cost and are included to illustrate that subjective responses parallel but do not duplicate precise calculations. The most important finding of Experiment 2 is that the relative ratings of Experiment 2 (Figure 4) are similar in pattern to the cost estimates (Figure 3) but are different from the relative ratings of Experiment 1 (Figure 2). A multiplicative model closely approximates the data for both parts of Experiment 2. A reasonable assumption is that subjects converted cost estimates to relative ratings through a linear transformation.

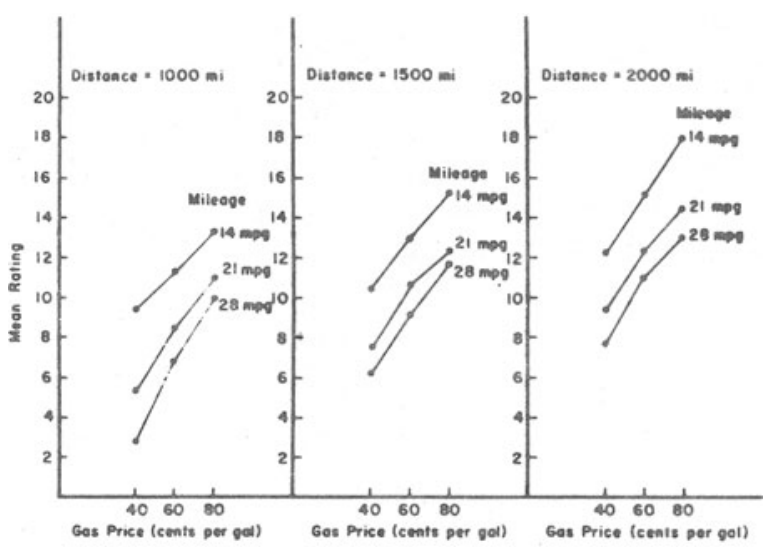

Figure 2. Mean ratings of relative expense for Experiment 1. (Higher numbers represent more expensive ratings.) 


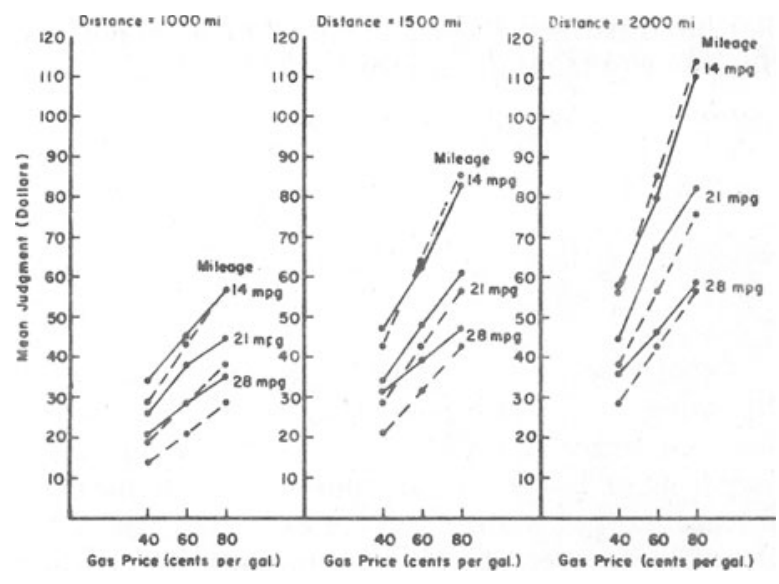

Figure 3. Mean numerical judgments (solid lines) and arithmetically correct values (dotted lines) of travel cost for Part 1 of Experiment 2.

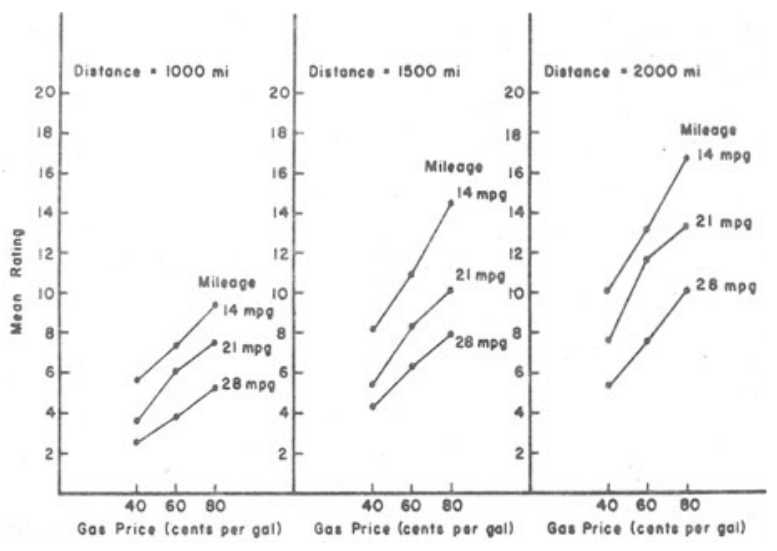

Figure 4. Mean ratings of relative expense for Part 2 of Experiment 2.

\section{CONCLUSIONS}

Support for a linear transformation in Experiment 2 suggests that the differences between the cost estimates and the relative ratings in Experiment 1 reflect different underlying integration processes rather than response transformation effects. An additive process for the relative ratings group could be interpreted to mean that these subjects, in the absence of an accuracy criterion for their responses chose the simplest (nonconfigural) rule for combining the information values (see also Shanteau \& Anderson, 1972).

This study illustrated that the inclusion of an overt response transformation condition can facilitate the interpretation of data from tasks where the response transformations are unobservable. In the present case, this technique showed that different integration processes are operative in nominally similar tasks when response requirements are varied. Furthermore, it provides support for one of the basic, but ordinarily untested, assumptions of information integration theory applied to subjective ratings, namely, that each piece of information is evaluated along the dimension of judgment prior to the integration process.

\section{REFERENCE NOTE}

1. Hensley, V. S., \& Levin, I. P. Judgments of travel expense: Application of models of information integration. Paper presented at the meeting of the Iowa Academy of Science, Ames, Iowa, April, 1975.

\section{REFERENCES}

Anderson, N. H. Application of an additive model to impression formation. Science, 1962, 138, 817-818.

ANDERson, N. H. Cross-task validation of functional measurement. Perception \& Psychophysics, 1972, 12, 389-395.

ANDERSON, N. H. Information integration theory: A brief survey. In D. H. Krantz, R. C. Atkinson, R. D. Luce, \& P. Suppes (Eds.), Contemporary developments in mathematical psychology (Vol. 2). San Francisco: Freeman, 1974.

Birnbaum, M. H. The nonadditivity of personality impressions. Journal of Experimental Psychology Monograph, 1974, 102, 543-561.

Birnbaum, M. H., \& Veit, C. T. Scale convergence as a criterion for rescaling: Information integration with difference, ratio, and averaging tasks. Perception \& Psychophysics, 1974, 15, 1-9.

Graesser, C. C. \& Anderson, N. H. Cognitive algebra of the equation: Gift size $=$ generosity $\times$ income. Journal of Experimental Psychology, 1974, 103, 692-699.

LEVIN, I. P. Information integration in numerical judgments and decision processes. Journal of Experimental Psychology: General, 1975, 104, 39-53.

Shanteau, J. C., \& ANDERson, N. H. Integration theory applied to judgments of the value of information. Journal of Experimental Psychology, 1972, 92, 266-275.

(Received for publication October 6, 1975.) 